\title{
Original Article (short paper) \\ Effect of aerobic exercise training on cGMP levels and blood pressure in treated hypertensive postmenopausal women
}

\author{
Iane P Novais \\ Universidade Estadual Paulista "Júlio de Mesquita Filho", Rio Claro, SP, Brasil \\ Universidade Estadual do Sudoeste da Bahia, Jequié, Bahia, Brasil \\ Aline P Jarrete \\ Guilherme M Puga \\ Hygor N Araujo \\ Universidade Estadual Paulista "Júlio de Mesquita Filho", Rio Claro, SP, Brasil \\ Maria A Delbin \\ Universidade Estadual de Campinas, Campinas, SP, Brasil \\ Angelina Zanesco \\ Universidade Estadual Paulista "Júlio de Mesquita Filho", Rio Claro, SP, Brasil \\ Universidade "Camilo Castelo Branco", Fernandópolis, SP, Brasil
}

\begin{abstract}
The second messenger cGMP has been largely studied as a therapeutic target in a variety of disorders such as erectile dysfunction, arterial hypertension and heart failure. Evidence has shown that cGMP activators are less efficient in estrogen-deficiency animals, but no studies exist involving non-pharmacological approaches on NO/ cGMP signaling pathway in hypertensive postmenopausal women. The aim of this study is to examine NO/cGMP pathway, redox state and blood pressure in trained treated hypertensive (HT) postmenopausal women comparing with normotensive (NT) group. The rationale for that is most of HT patients is encouraged by physician to perform exercise associated with pharmacological treatments. Aerobic exercise training (AET) consisted of 24 sessions, 3 times/week. Parameters were evaluated at baseline and after AET for both groups ( $\mathrm{HT}=28$; NT=33).In treated HT group, AET was significantly effective in increasing cGMP concentrations (28\%) accompanied by an up-regulation of SOD (97\%) and catalase activity (37\%). In NT group, we found an increase in SOD activity $(58 \%)$. Treated HT postmenopausal women were still responsive to AET increasing cGMP levels and up-regulating antioxidant system. It should also be emphasized that these findings provide information on the circulating biomarkers that might delay the developing of cardiovascular events in this particular population.
\end{abstract}

Keywords: hypertension;postmenopause;aerobic exercise, cGMP, redox state.

\section{Introduction}

The second messenger cyclic guanosine 3'5'monophosphate (cGMP) has been largely studied as a therapeutic target in a variety of disorders such as erectile dysfunction, arterial hypertension, atherosclerosis and heart failure. Indeed, agents that increase cGMP generation or decrease its degradation,such as inhibitors of phosphodiesterase 5 (PDE5) and activators of soluble guanylylcyclase, have been investigated by different laboratories $^{1,2}$. Strategies to enhance cGMPlevels would be useful in management cardiovascular diseases (CVD) or in preventing their complications. Of note, it was recently demonstrated that the efficacy of PDE5 inhibition in cardiac diseases is dependent of estrogen levels in female mice ${ }^{3}$. Thus, clinical trials examining some biomarkers in women after menopause are extremely relevant.

It has been shown that aerobic exercise training (AET) is an important approach to prevent or to mitigate the complications of
CVD promoting an improvement of eNOS/NO signaling pathway in both humans and laboratory animals ${ }^{4,5}$. Interestingly, only two studies exist evaluating the effects of AET on cGMP levels in women after menopause; however, the number of the participants were too small and no age-matched group was studied ${ }^{6,7}$. The objective of this study was to examine the effects of AET on cGMP levels in hypertensive postmenopausal women treated with antihypertensive therapy. The rationale for that is most of hypertensive patients is encouraged by physician to perform physical exercise associated with pharmacological treatments. Therefore, it would be feasible to evaluate treated participants in an attempting to detect additional effects of both interventions (AET plus antihypertensive drugs). We also compared treated hypertensive postmenopausal women with normotensive and the effects of AET on blood pressure and its association with cardiovascular biomarkers. 


\section{Materials and Methods}

\section{Participants}

Sixty-one postmenopausal women were enrolled in the study, normotensive group (NT, $\mathrm{n}=33$ ) and treated hypertensive group(HT, $\mathrm{N}=28$ ) according to previous medical diagnosis. The inclusion criteria for the participants were: reported absence of menses for at least 1 year at recruitment, body mass index $\leq 30$ $\mathrm{kg} / \mathrm{m}^{2}$, and physically inactivity $(<150$ minutes of moderate physical activity per week or $<60$ minutes of vigorous physical activity per week). Exclusion criteria were: menopausal hormone therapy use, smoking, individuals with diabetes (type 1 or 2), previous CVD (stroke, heart failure), renal dysfunction, inability to perform physical exercise, poorly controlled hypertension ${ }^{8}$.

The study was approved by the Ethical Committee of the Bioscience Institute of the University of São Paulo State (Ref.4395/2010) and each participant signed a written informed consent.

\section{Experimental Design}

Participants of the study underwent to 24-session of AET, see Figure 1 for more details. Anthropometric assessment (height, weight, body mass index), aerobic capacity test, biochemical analyses were performed at baseline and after AET. The participants were instructed to maintain their habitual diet routine during the protocol.

\section{Anthropometric and cardiovascular assessments}

Body weight $(\mathrm{kg})$ and height $(\mathrm{cm})$ were determined using a scale and stadiometer (Toledo ${ }^{\circledR} 2096 \mathrm{PP}$ ) and body mass index was calculated (BMI in $\mathrm{kg} / \mathrm{m}^{2}$ ). Blood pressure (BP) was performed using a semi-automatic equipment (Microlife ${ }^{\circledR}$ MIB-P3BTOA) and three BP measurements were taken. Resting BP was determined as the average of the measurements. Heart rate (HR) was assessed using a heart rate monitor (Polar ${ }^{\circledR}$ FT1 TRQ). Both BP and HR were evaluated after 20 minutes in the sitting position. All measurements were taken following the Brazilian Guidelines of Hypertension?

\section{Aerobic Capacity Test}

Before the exercise tests and training program, women were familiarized to the treadmill by walking during 2-3 days, depending on each participant.After that, all participants performed 2-5 tests to determine the maximal lactate steady state (MLSS)to be used for the intensity of AET prescription. Each constant workload test with fixed walking speed $(5.5 \mathrm{Km} / \mathrm{h})$ on a treadmill (Movement ${ }^{\circledR}$ RT 250 PRO) lasted 30 min according to previous study ${ }^{10}$. The exercise intensity was controlled by the inclination of the treadmill (measured in \%), with the grade adjusted according to the aerobic capacity of the participant in each session. The inclination ranged between $2 \%$ and $10 \%$ during the test sessions. Capillary blood samples $(25 \mu \mathrm{L})$ were collected by micropuncture(earlobe) at tenth and thirtieth minutes for the determination of blood lactate concentration $([\mathrm{Lac}])^{11}$. The MLSS workload (anaerobic threshold) was defined as the highest power intensity(treadmill inclination) at which blood lactate concentration did not increase by more than $1 \mathrm{nmol} / \mathrm{L}$ between the tenth and thirtieth minutes of the constant load test ${ }^{12}$.

\section{Blood collection and biochemical analyses}

Blood samples were collected at $07 \mathrm{~h} 30 \mathrm{a} . \mathrm{m}$. in two different days: after 12 hours of overnight fast (fasting) and after daily breakfast (postprandial). Blood samples were immediately centrifuged at $3000 \mathrm{rpm}$ during $10 \mathrm{~min}$, and the supernatant (plasma and serum) was separated into several aliquots and stored at $-80^{\circ} \mathrm{C}$ for biochemical analyses.

\section{Nitrite/Nitrate $\left(N O_{x}^{-}\right)$and cGMPlevels}

Plasma concentrations of $\mathrm{NO}_{x}^{-}$were measured to evaluate nitric oxide (NO) production by commercial available kit (Cayman Chemical $^{\circledR}$, Ann Arbor, MI, USA). Before starting this assay, samples were ultra-filtered through micro filter (Microcon ${ }^{\circledR}$ Centrifugal Filter Units, $10 \mathrm{kDa}$, Millipore, Bedford, MA). Plasma concentrations of cGMP were measured by ELISA method using a commercial available kit (Cayman Chemical ${ }^{\mathbb{} \text {, }}$ Ann Arbor, MI, USA).

\begin{tabular}{|c|c|c|}
\hline 2 Weeks & 8 Weeks & 2 Weeks \\
\hline BASELINE & EXERCISE TRAINING & POST AET \\
\hline 1. Anthropometric Assessment & 24 sessions $\mid 30-40 \mathrm{~min}$ & 1. Anthropometric Assessment \\
\hline 2. Aerobic Capacity Test & 3 days/week & 2. Aerobic Capacity Test \\
\hline 3. Fasting and Postprandial Exam & At anaerobic threshold intensity & 3. Fasting and Postprandial Exam \\
\hline
\end{tabular}

Figure 1.Study design. 


\section{Antioxidant enzyme activities and lipid peroxidation}

Superoxide Dismutase (SOD) and catalase (CAT) activity were measured by ELISA method using a commercial available kit (Cayman $\mathrm{Chemical}^{\circledR}$, Ann Arbor, MI, USA). SOD’s assay detects radicals superoxide generated by xanthine oxidase and hypoxanthine, revealing the plasmatic activity of this enzyme. Catalase's assay is based on the reaction of the enzyme with methanol in an optimal $\mathrm{H}_{2} \mathrm{O}_{2}$ concentration. Levels of Thiobarbituric Acid Reactive Substances (TBARS) were determined by using the TBARS assay kit again from Cayman Chemical ${ }^{\circledR}$ (Ann Arbor, MI, USA) in which the MDA-TBA adduct formed by the reaction of malondialdehyde (MDA) and thiobarbituric acid (TBA) under high temperature $\left(90-100^{\circ} \mathrm{C}\right)$ and acidic conditions is measured colorimetrically at $530-540 \mathrm{~nm}$. This method reflects lipid peroxidation and was expressed in $\mu \mathrm{M}$ MDA.

\section{Aerobic exercise training (AET)}

The AET program began with appropriate warm up $(5 \mathrm{~min}$ speed walking $4 \mathrm{~km} / \mathrm{h}$ on a treadmill without inclination) under supervision. Participants performed the exercise session on a treadmill in a quiet room with environment-controlled temperature $\left(\gg 25^{\circ} \mathrm{C}\right)$ and humidity $(» 50 \%)$. Physical exercise was performed 3 days/week, for a total of 8 weeks (24 sessions), and each session consisting of $30 \mathrm{~min}$ (first 3 weeks), $35 \mathrm{~min}$ (following 3 weeks), and 40 min (last 2 weeks). The intensity (treadmill inclination) was prescribed according to previous individual MLSS test. Heart rate and the rate of perceived exertion (Borg's scale) were also recorded every $10 \mathrm{~min}$ in each session.

\section{Statistical analysis}

Data are presented as mean \pm standard error mean. Before applying statistical methods, the normality of the data was tested by the Kolmogorov-Smirnov's test. Two-way analysis of variance (ANOVA) was used comparing the effect of AET and difference between groups (NT and HT). Bonferroni correction was used as a post hoc test. The power of the study was $95 \%$ for both groups. Statistical analysis was performed using the GraphpadPrism ${ }^{\circledR}$ version 5 software package (California, USA), and the level of significance was set at $\mathrm{p}<0.05$.

\section{Results}

Both groups were homogenous in terms of time after menopause (NT: $8.0 \pm 0.9 ;$ HT: $10.3 \pm 1.2 \mathrm{yrs}$ ). Approximately $70 \%$ of treated hypertensive women were on angiotensin system inhibition plus diuretics and $30 \%$ were on beta blocker/calcium channel blocker.

Anthropometric and cardiovascular parameters, lipid profile, and blood glucose were similar between NT and HT postmenopausal women at baseline. Exercise training did not modify these parameters(Table 1).

Table 1. Effects of aerobic exercise training on anthropometric, cardiovascular, biochemical parameters and aerobic capacity test in hypertensive postmenopausal women comparing with normotensive.

\begin{tabular}{|c|c|c|c|c|}
\hline & \multicolumn{2}{|c|}{ NT $(n=33)$} & \multicolumn{2}{|c|}{ HT $(n=28)$} \\
\hline & Baseline & Post AET & Baseline & Post AET \\
\hline Age (years) & $55.6 \pm 0.8$ & & $57.2 \pm 1.0$ & \\
\hline Body Mass Index $\left(\mathrm{kg} / \mathrm{m}^{2}\right)$ & $27.1 \pm 0.6$ & $27.1 \pm 0.6$ & $28.2 \pm 0.5$ & $28.0 \pm 0.5$ \\
\hline Systolic BP (mmHg) & $113.6 \pm 2.0$ & $112.6 \pm 2.1$ & $117.3 \pm 2.0$ & $115.3 \pm 2.1$ \\
\hline Diastolic BP (mmHg) & $70.4 \pm 1.4$ & $69.7 \pm 1.3$ & $73.2 \pm 1.8$ & $69.9 \pm 2.0$ \\
\hline Heart Rate (bpm) & $72.7 \pm 1.6$ & $69.7 \pm 1.8$ & $69.3 \pm 1.9$ & $67.7 \pm 1.8$ \\
\hline Total Cholesterol (mg/dl) & $210.0 \pm 5.2$ & $204.3 \pm 4.6$ & $199.4 \pm 5.8$ & $189.7 \pm 4.5$ \\
\hline C-HDL (mg/dl) & $45.6 \pm 1.6$ & $48.3 \pm 1.4$ & $47.4 \pm 1.3$ & $48.3 \pm 1.2$ \\
\hline C-LDL (mg/dl) & $137.0 \pm 5.5$ & $130.6 \pm 4.3$ & $126.9 \pm 5.7$ & $119.3 \pm 4.4$ \\
\hline C-VLDL (mg/dl) & $26.5 \pm 2.1$ & $25.5 \pm 1.7$ & $25.1 \pm 1.6$ & $22.0 \pm 1.4$ \\
\hline Triglycerides (mg/dl) & $132.5 \pm 10.5$ & $127.5 \pm 8.6$ & $125.6 \pm 8.1$ & $110.0 \pm 7.2$ \\
\hline Blood glucose (mg/dl) & $87.3 \pm 1.6$ & $90.8 \pm 1.6$ & $88.0 \pm 1.6$ & $90.7 \pm 1.9$ \\
\hline Intensity (treadmill inclination \%) & $5.7 \pm 0.4$ & $7.9 \pm 0.5^{\mathrm{a}}$ & $5.0 \pm 0.4$ & $6.8 \pm 0.4^{\mathrm{a}}$ \\
\hline$[\mathrm{Lac}]$ at Anaerobic Threshold $(\mu \mathrm{M})$ & $2.9 \pm 0.2$ & $3.1 \pm 0.2$ & $3.3 \pm 0.3$ & $3.0 \pm 0.3$ \\
\hline
\end{tabular}

Abbreviations: $\mathrm{BP}$ - Blood pressure; C-HDL - high-density lipoprotein cholesterol; C-LDL - low-density lipoprotein cholesterol; C-VLDL - very low-density lipoprotein cholesterol; [Lac] - Blood lactate concentration.

a Significantly different compared with respectively baseline $(p<0.05)$. 
Exercise training for 24 sessions was effective in increasing the exercise intensity without changes in blood lactate concentration, approximately $38 \%$ and $35 \%$ for NT and HT women, respectively, showing the efficacy of the AET in improving aerobic capacity.

Given that experimental studies have attributed to the greater prevalence of arterial hypertension in women after menopause to endothelial dysfunction, we evaluated NO/cGMP signaling pathway in both groups. We found no differences in the concentrations of $\mathrm{NO}_{\mathrm{x}}^{-}$and cGMP between NT and HT women at baseline. In fasting state, a marked increase in the concentration of $\mathrm{NO}_{\mathrm{x}}$ - was found in trained HT group, approximately $42 \%$, as compared with trained NT group. At postprandial state, trained hypertensive group had an increase in cGMP concentration $(28 \%)$, but no changes in $\mathrm{NO}_{\mathrm{x}}^{-}$levels were observed. Figure 2 illustrates these data.

Figure 2.Effects of aerobic exercise training on NOx- and cGMP in normotensive and hypertensive postmenopausal women at baseline and post aerobic exercise training.
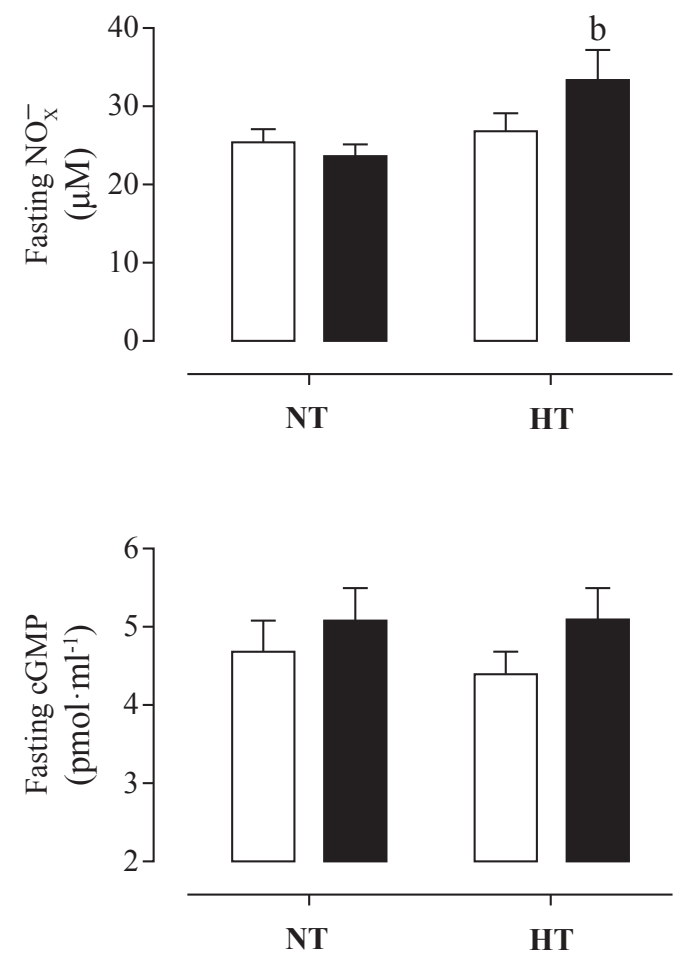

a Significantly different as compared with its respectively baseline $(\mathrm{p}<0.05)$.

${ }^{\mathrm{b}}$ Significantly different as compared with post AET NT group $(\mathrm{p}<0.05)$.

We also examined fasting redox state by measuring TBARS concentration and antioxidant enzymes in plasma. At baseline, no differences were found between the groups and exercise training did not change TBARS concentration that reflects lipid peroxidation. In contrast, SOD activity was significantly
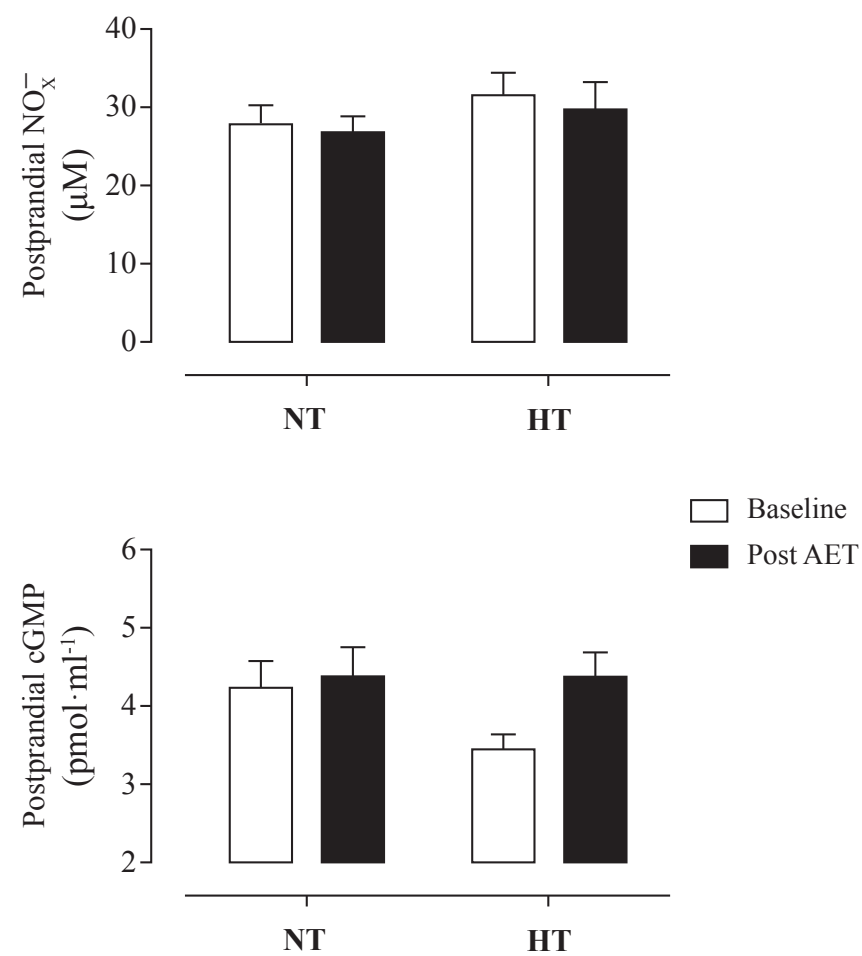

increased in both groups (58 and 97\% for NT and HT women, respectively). Moreover, trained HT group presented higher SOD activity as compared with NT women, approximately $27 \%$. Interestingly, catalase activity was significantly increased in HT group, approximately $73 \%$ (Figure 3 ).

Figure 3. Fasting redox state in normotensive and hypertensive postmenopausal women at baseline and post aerobic exercise training.
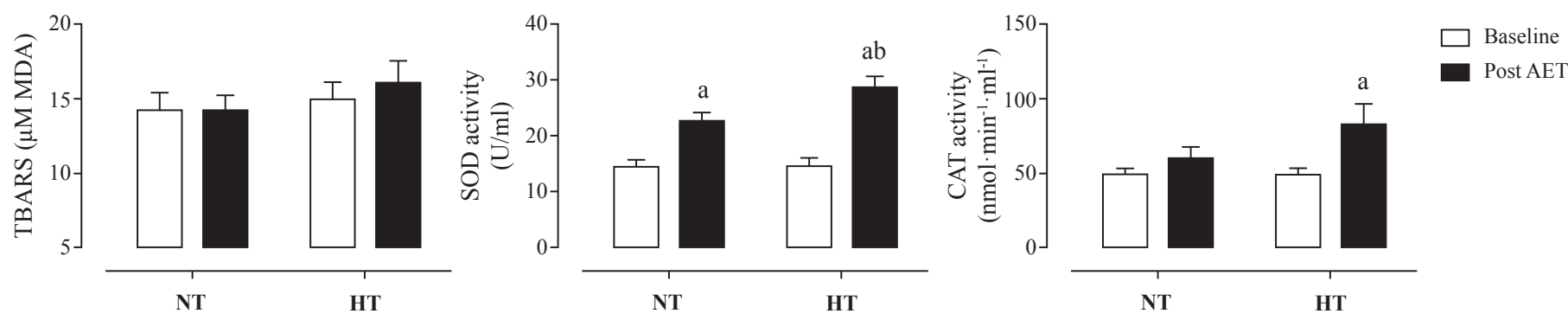

a Significantly different as compared with its baseline $(\mathrm{p}<0.05)$.

b Significantly different as compared with post AET NT group $(\mathrm{p}<0.05)$ 


\section{Discussion}

Our findings show that treated HT postmenopausal women are still responsive to AET with increase in cGMP levels and up-regulation of the antioxidant system even though BP was well-controlled.

Arterial hypertension is a chronic disease that may lead to a variety of complications such as vascular remodeling, heart attack, stroke and heart failure ${ }^{13}$. The Global Burden of Disease Study identified that uncontrolled BP is the leading risk factor for death and disability-adjusted life-years $\operatorname{lost}^{14}$. Moreover, it has shown that the incidence of CVD in women increases after menopause ${ }^{15}$. It is believed that sex hormones play a major role and estrogen deficiency might be the primary cause of this phenomenon in women in climacteric period; however, most of studies are based on experimental studies, using ovariectomized animals or isolated cells ${ }^{16}$.

Although no changes in blood pressure values were found in trained HT group, AET was effective in promoting increase in cardiovascular biomarkers that play a crucial role on BP regulation or oxidative stress modulation. Accordingly, it has been demonstrated that exercise training is more effective in lowering blood pressure in uncontrolled hypertensive subjects ${ }^{17}$. Moreover, epidemiological studies have systematically reported that higher fitness levels could delay the developing of arterial hypertension or its complications, showing the beneficial effects of physical activity/exercise on $\mathrm{CVD}^{18,19}$. Thus, our findings are clinically relevant since most of HT patients are encouraged to get involved in physical activities or in exercise training under supervision.

Of note, great interest exists regarding new pharmacological targets to increase cGMPlevels, either increasing cGMP generation or decreasing its degradation mainly in patients with heart failure ${ }^{2}$. Nevertheless, recent clinical data were disappointing mainly because of up-regulation of PDE5 during long-term inhibition treatment ${ }^{20}$. Interestingly, our study shows that HT postmenopausal women are responsive in increasing cGMP levels after AET that would prevent or, so far, delay the developing of heart failure during climacteric period.

Only one study evaluated postmenopausal women and found no differences in cGMP levels between the control group and patients with unstable coronary artery disease (CAD) (Nielsen et al, 1999). Indeed, patients with signs of ischaemia in the form of ST-depression during an exercise test had higher levels of cGMP than those without signs of ischaemia. Another study found no differences in basal plasma cGMP levels between healthy physically active, healthy inactive, and in patients with coronary artery disease, however, short-term aerobic exercise increased cGMP urinary concentrations in healthy subjects and in CAD patients, if measured during and soon after termination of the exercise (Alfieri et al, 2000). Nevertheless, the participants were men up to 49 years old and there is no data available in hypertensive postmenopausal women.

The possible mechanism for that may be related to the effect of shear stress induced by physical exercise. Indeed, physical exercise is a powerful stimulus to promote vascular shear stress activating mechanosensors present in endothelial cells. These mechanosensors are coupled to complex biochemical signaling pathways, such as Ras/MEK/ERK, c-Src, G proteins, ion channel, VE-cadherin, and PI3K/Akt, which in turn, regulate $\mathrm{NO} /$ cGMP pathway ${ }^{21}$. Accordingly, the beneficial effects of physical training on cardiovascular system are strongly associated with increased blood flow 4 .

Differently from experimental studies, no difference was found in TBARS levels and antioxidant enzymes activity between the two groups at baseline. Indeed, few studies exist examining this issue in human population. A previous study showed an increase in oxidative stress in obese postmenopausal women $^{22}$ whereas another study demonstrated a decrease in antioxidant defense in older population as compared to young in healthy women and men $^{23}$. Regarding the effect of physical exercise, we found an up-regulation of SOD activity in both groups and this effect was greater in hypertensive group. Accordingly, a cross-sectional study evaluating postmenopausal women demonstrated increased total SOD, cytosolic SOD and CAT activities in physically active group in comparison to sedentary ${ }^{24}$. Another study reported increased SOD activity in overweight and obese postmenopausal women after a 4-month program of 1-hour low- intensity physical activity for 2 days per week $^{25}$. Collectively, AET is an important approach to promote up-regulation antioxidant enzymes in postmenopausal women that might result in beneficial effect in cardiovascular system. However, more studies are necessary to examine the exerciserelated oxidative stress in this population in an attempt to provide new approaches for health care and preventing the deleterious effects of CVD in women.

\section{Conclusion}

In conclusion, our findings show that AET is an effective nonpharmacological approach in increasing cGMP levels as well as up-regulating antioxidant enzymes in treated HT postmenopausal women. It should also be emphasized that these findings provide information on the circulating biomarkers that might delay the developing of cardiovascular events in this population.

\section{References}

1. Stasch JP, Pacher P, Evgenov OV. Soluble guanylate cyclase as an emerging therapeutic target in cardiopulmonary disease. Circulation. 2011;24;123(20):2263-73.

2. Buglioni A, Burnett JC Jr. New Pharmacological Strategies to Increase cGMP. Annu Rev Med.2016;67:229-43.

3. Sasaki H, Nagayama T, Blanton RM, Seo K, Zhang M, Zhu G, et al. PDE5 inhibitor efficacy is estrogen dependent in female heart disease. J Clin Invest. 2014;124(6):2464-71.

4. Zanesco A, Antunes E. Effects of exercise training on the cardiovascular system: pharmacological approaches. Pharmacol Ther. 2007;114(3):307-17.

5. Claudino MA, Franco-Penteado CF, Priviero FB, Camargo EA, Teixeira SA, Muscará MN, et al. Upregulation of gp91phox subunit of $\mathrm{NAD}(\mathrm{P}) \mathrm{H}$ oxidase contributes to erectile dysfunction 
caused by long-term nitric oxide inhibition in rats: reversion by regular physical training. Urology. 2010;75(4):961-7.

6. Maeda S, Tanabe T, Otsuki T, Sugawara J, Iemitsu M, Miyauchi T, et al. Moderate regular exercise increases basal production of nitric oxide in elderly women. Hypertens Res. 2004;27(12):947-53.

7. Jarrete AP, Novais IP, Nunes HA, Puga GM, Delbin MA, Zanesco A. Influence of aerobic exercise training on cardiovascular and endocrine-inflammatory biomarkers in hypertensive postmenopausal women. J Clin Transl Endocrinol. 2014;1(3):108-14.

8. James PA, Oparil S, Carter BL, Cushman WC, DennisonHimmelfarb C, Handler J, et al. 2014 evidence-based guideline for the management of high blood pressure in adults: report from the panel members appointed to the Eighth Joint National Committee (JNC 8). JAMA. 2014; 311: 507-20.

9. Sociedade Brasileira de Cardiologia. [VII Brazilian Guidelines on Hypertension]. Arq Bras Cardiol. 2016;107(3).

10. Benecke R. Anaerobic threshold, individual anaerobic threshold, and maximal lactate steady state in rowing. Med Sci Sports Exerc. 1995;27(6):863-7.

11. Engel PC, Jones JB. Causes and elimination of erratic blanks in enzymatic metabolite assays involving the use of NAD + in alkaline hydrazine buffers: improved conditions for the assay of L-glutamate, L-lactate, and other metabolites. Anal Biochem. 1978;88(2):475-84.

12. Beneke R Methodological aspects of maximal lactate steady state-implications for performance testing. Eur J Appl Physiol. 2003;89(1):95-9.

13. Borlaug BA, Paulus WJ. Heart failure with preserved ejection fraction: pathophysiology, diagnosis, and treatment. Eur Heart J. 2011;32(6):670-9.

14. Lim SS, Vos T, Flaxman AD, Danaei G, Shibuya K, Adair-Rohani $\mathrm{H}$, et al. A comparative risk assessment of burden of disease and injury attributable to 67 risk factors and risk factor clusters in 21 regions, 1990-2010: a systematic analysis for the Global Burden of Disease Study 2010. Lancet. 2001;380:2224-60.

15. Mozaffarian D, Benjamin EJ, Go AS, Arnett DK, Blaha MJ, Cushman M, et al. Heart disease and stroke statistics-2015 update: a report from the American Heart Association. Circulation. 2015.27;131(4):e29-322.

16. Maranon R, Reckelhoff JF. Sex and gender differences in control of blood pressure. Clin Sci (Lond) 2013;125:311-18.

17. Fagard RH. Exercise is good for your blood pressure: effects of endurance training and resistance training. Clin Exp Pharmacol Physiol. 2006;33(9):853-6.

18. Blair SN, Kohl HW 3rd, Paffenbarger RS Jr, Clark DG, Cooper KH, Gibbons LW. Physical fitness and all-cause mortality.
A prospective study of healthy men and women. JAMA. 1989;262(17):2395-401.

19. Liu J, Sui X, Lavie CJ, Zhou H, Park YM, Cai B, et al. Effects of cardiorespiratory fitness on blood pressure trajectory with aging in a cohort of healthy men. J Am Coll Cardiol. 2014;64(12):1245-53.

20. Redfield MM, Chen HH, Borlaug BA, Semigran MJ, Lee $\mathrm{KL}$, Lewis G, et al. Effect of phosphodiesterase-5 inhibition on exercise capacity and clinical status in heart failure with preserved ejection fraction: a randomized clinical trial. JAMA. 2013;309(12):1268-77.

21. Balligand JL, Feron O, Dessy C. eNOS activation by physical forces: from short-term regulation of contraction to chronic remodeling of cardiovascular tissues. Physiol Rev. 2009;89(2):481-534.

22. Uppoor RB, Rajesh A, Srinivasan MP, Unnikrishnan B, Holla R. Oxidative Stress in Obese Postmenopausal Women: An Additive Burden for Atherosclerosis. J Clin Diagn Res. 2015;9(12):OC03-OC05.

23. Singh K, Kaur S, Kumari K, Singh G, Kaur A. Alterations in Lipid Peroxidation and Certain Antioxidant Enzymes in Different Age Groups under Physiological Conditions. J Hum Ecol. 2009;27(2):143-147.

24. Farinha JB, De Carvalho NR, Steckling FM, De Vargas Lda S, Courtes AA, Stefanello ST, et al. An active lifestyle induces positive antioxidant enzyme modulation in peripheral blood mononuclear cells of overweight/obese postmenopausal women. Life Sci. 2015;121:152-7.

25. Merino J, Ferré R, Girona J, Aguas D, Cabré A, Plana N, et al. Even low physical activity levels improve vascular function in overweight and obese postmenopausal women. Menopause. 2013;20(10):1036-42.

\section{Corresponding author}

Iane de Paiva Novais, Assistant Professor

Health Department, State University of Southwestern Bahia - UESB

Address: Avenida Jose Moreira Sobrinho s/n, Jequiezinho

Jequie-BA/Brazil.

Email: ianepaiva@yahoo.com.br

Manuscript received on December 11, 2016

Manuscript accepted on December 12, 2016

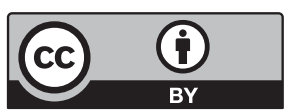

Motriz. The Journal of Physical Education. UNESP. Rio Claro, SP, Brazil - eISSN: 1980-6574 - under a license Creative Commons - Version 3.0 